\title{
AVALIAÇÃO FUNCIONAL DE IDOSOS COM AMPUTAÇÃO DE MEMBROS INFERIORES ATENDIDOS EM UM HOSPITAL UNIVERSITÁRIO'
}

Maria José D'Elboux Diogo ${ }^{2}$

Diogo MJD. Avaliação funcional de idosos com amputação de membros inferiores atendidos em um hospital universitário. Rev Latino-am Enfermagem 2003 janeiro-fevereiro; 11(1):59-65.

Foram entrevistados 40 idosos com amputação acima dos maléolos em acompanhamento no ambulatório de Órteses e Próteses do Hospital das Clínicas da Universidade Estadual de Campinas, no período de junho de 1994 a junho de 1999, com objetivo de avaliar o nível de independência, segundo o índice de Barthel, e de identificar relações entre o índice de Barthel e o tipo e nível da amputação, e uso da prótese. Os sujeitos desta pesquisa apresentam elevado grau de independência para as atividades da vida diária, segundo o índice de Barthel. Houve associação significativa somente entre o índice de Barthel e o tipo de amputação, indicando que os idosos com amputação unilateral são mais independentes do que aqueles com amputação bilateral.

DESCRITORES: reabilitação, amputação, geriatria

\section{FUNCTIONAL EVALUATION OF ELDERLY WITH LOWER LIMB AMPUTATION FOLLOWED AT A UNIVERSITY HOSPITAL}

We studied 40 elderly with lower limb amputation (above the malleolus), who attended a hospital outpatient clinic (Orthoses and Prostheses Unity, University Hospital, State University of Campinas), from June 1994 to June 1999. Our objectives were to evaluate the independence level according to the Barthel's Index and to identify relationships between the mentioned Index and the type and level of amputation, as well as the prosthetic use. The subjects had a high degree of independence, according to the Barthel's Index. We found statistical significance only for the relationship between the Barthel's Index and the type of amputation, suggesting that the elderly with unilateral amputation were more independent than those who had undergone bilateral amputation.

DESCRIPTORS: rehabilitation, amputation, geriatrics

\section{EVALUACIÓN FUNCIONAL DE ANCIANOS CON AMPUTACIÓN DE LOS MIEMBROS INFERIORES ATENDIDOS EN UN HOSPITAL UNIVERSITARIO}

Fueron entrevistados 40 ancianos con amputación por encima de los maleolos, que estaban en seguimiento ambulatorio en la Unidad de Órtesis y Prótesis del Hospital Clínicas de la Universidad Estatal de Campinas, en el período de junio de 1994 a junio de 1999. El objetivo fue evaluar el nivel de independencia según el Índice de Barthel e identificar las relaciones entre el Índice de Barthel, el nivel de amputación, y el uso de prótesis. Los sujetos de esta investigación presentaron elevado grado de independencia para las actividades de la vida diaria según el Índice de Barthel. El análisis mostró asociación significativa apenas entre el Índice de Barthel y el tipo de amputación, indicando que los ancianos con amputación unilateral son mas independientes que aquellos con amputación bilateral.

DESCRIPTORES: rehabilitación, amputación, geriatría

\footnotetext{
Artigo elaborado com dados da tese de Livre Docência apresentada no Departamento de Enfermagem da Faculdade de Ciências Médicas da Universidade Estadual de Campinas. FAPESP Proc. №00/05537-2; ${ }^{2}$ Enfermeira, Professor Associado no Departamento de Enfermagem da Faculdade de Ciências Médicas da Universidade Estadual de Campinas, e-mail: mariadio@uol.com.br
} 
INTRODUÇÃO

$\boldsymbol{A}$ reabilitação de idosos com amputação de membros inferiores é um tema ainda pouco investigado em nosso meio. Em âmbito internacional, a maioria dos trabalhos sobre a reabilitação dessas pessoas é descritivo e retrospectivo, e busca relacionar diferentes variáveis, tais como idade, causa e nível da amputação, comorbidade e dependência funcional ${ }^{(1)}$.

A idade, por si só, como critério para a indicação do uso de prótese, é um ageísmo importante ${ }^{(2)}$. Concordamos com esses autores e acrescentamos que, não somente a indicação de uma prótese, mas investimentos, sejam de natureza assistenciais, sociais e educativas, direcionados à reabilitação de idosos, também sofrem a influência do ageísmo, perpetuando o mito de que pessoas idosas têm menor potencial para a reabilitação. Não podemos generalizar, pois os idosos constituem grupo heterogêneo, cujas características individuais devem ser consideradas na reabilitação.

Sobre o nível da amputação, raramente os estudos sobre o tema especificam o uso de métodos para determinar o nível da cirurgia ${ }^{(1)}$. Porém, cabe a atenção para alguns aspectos referentes à avaliação pré-operatória, entre eles a avaliação global do paciente, condições física e social, a viabilidade do membro e condições da pele, a capacidade física, expectativas, adequações necessárias no domicílio e rede de suporte para o cuidado ${ }^{(3)}$.

$\mathrm{Na}$ realidade, quando entendemos reabilitação como processo dinâmico, criativo, progressivo e educativo, cujos objetivos estão direcionados à restauração funcional ótima do indivíduo, sua reintegração à família, à comunidade e à sociedade, por meio da máxima independência nas atividades da vida diária, vários fatores necessitam ser considerados, entre eles a presença de múltiplas afecções, a independência funcional e a autonomia ${ }^{(3)}$.

A visão da sociedade sobre a pessoa portadora de deficiência é alterada drasticamente quando essa pessoa demonstra independência para as Atividades da Vida Diária (AVDs), pois vai de encontro ao preconceito social existente relacionado ao peso social e financeiro que o portador de deficiência acarreta para a sociedade. Isto é, uma vez demonstrando independência, o indivíduo apresenta capacidade ou potencial para exercer seu papel social e produtivo perante a sociedade. A incapacidade física pode ser uma barreira para a independência quando as pessoas assim a vêem ou quando o ambiente a propicia.
A avaliação da capacidade funcional na gerontologia é importante indicativo da qualidade de vida do idoso, sendo o desempenho nas atividades da vida diária o parâmetro amplamente aceito e reconhecido, pois permite aos profissionais e, em particular, à enfermeira uma visão mais precisa quanto à severidade da doença $\mathrm{e}$ das suas seqüelas. Assim, entendemos como avaliação funcional a designação dada para uma função específica, a capacidade de se autocuidar e de atender as necessidades básicas diárias, ou seja, as Atividades da Vida Diária (AVDs). Entre as clássicas AVDs propostas inicialmente por Sidney Katz, incluem-se a capacidade para alimentar-se, transferir-se, vestir-se, banhar-se, ter continência e usar o banheiro. Outras atividades como preparar as refeições, lavar roupa, cuidar da casa, fazer compras, usar o telefone, usar o transporte, controlar o dinheiro e os medicamentos são as Atividades Instrumentais da Vida Diária (AIVDs), propostas por Lawton e Brody ${ }^{(4-5)}$.

Considerando que a avaliação da capacidade funcional é importante indicativo da reabilitação de idosos com membros inferiores, e que o grau de dependência está associado ao tipo (unilateral ou bilateral) e nível da amputação (transtibial, transfemural e outros), bem como ao uso da prótese, o presente estudo teve os seguintes objetivos:

- avaliar o atual nível de independência dos idosos atendidos no ambulatório de órteses e próteses da UNICAMP para as atividades da vida diária, segundo 0 índice de Barthel;

- identificar relações entre o índice de Barthel e o tipo e nível da amputação, e uso da prótese.

\section{MÉTODO}

Este artigo é parte de uma investigação mais abrangente que foi realizada no ambulatório de amputados da Unidade de Órteses e Próteses (UOP) do Hospital das Clínicas da Universidade Estadual de Campinas, no município de Campinas, SP, após o consentimento da chefia da unidade e do Comitê de Ética em Pesquisa da instituição.

Os dados foram obtidos por meio da entrevista, utilizando-se do índice de Barthel ${ }^{(6)}$ para a avaliação da dependência e de questões referentes à amputação, tais como nível e tipo da amputação e uso da prótese. 
A amostra foi composta por 40 idosos (idade mínima de 60 anos), atendidos no ambulatório de amputados, no período compreendido entre junho de 1994 e junho de 1999, com amputação de membros inferiores acima dos maléolos, e de comum acordo em participar da pesquisa, segundo a Resolução 196/96 do Conselho Nacional de Saúde.

\section{RESULTADOS}

No que diz respeito ao tipo da amputação, 31 pacientes possuíam o tipo unilateral, e nove bilateral.

$\mathrm{Na}$ Tabela 1 verificamos que, dos idosos com amputação unilateral, 18 utilizavam prótese, ainda que, segundo as respostas obtidas, 12 deles a utilizavam de modo contínuo ou seja, retiravam-na somente para dormir ou tomar banho, permanecendo com ela em casa e para sair; seis idosos usavam a prótese de modo não contínuo, isto é, somente em casa, para sair ou alternando seu uso no decorrer do dia.

Já entre os idosos com amputação bilateral, apenas dois utilizavam o membro artificial em ambos os cotos, sendo que um deles, com amputação transtibial bilateral, usava as próteses de modo contínuo. O outro, com amputação transtibial esquerda e transfemural direita, utilizava-as de modo não contínuo (somente em casa).

Para as amputações acima do joelho a prótese mais comum foi a de apoio quadrilátero (cinco sujeitos). Cabe destacar que entre os idosos que não usavam o membro artificial, nesse nível de amputação, dois referiram a não adaptação a esse tipo de prótese. Para as amputações abaixo do joelho, 12 idosos utilizavam a prótese tipo PTB (Patella Tendon Bear, de origem americana), e dois, o tipo KBM (Kondylen Bettung Münster, de origem alemã).

Tabela 1 - Distribuição dos idosos, segundo o índice de Barthel e o tipo de amputação e uso da prótese. Campinas-SP, 2000

\begin{tabular}{|c|c|c|c|c|c|c|}
\hline \multirow[t]{2}{*}{$\begin{array}{c}\text { Índice de } \\
\text { Barthel }\end{array}$} & \multicolumn{3}{|c|}{$\begin{array}{c}\text { Amputação Unilateral } \\
\text { Uso da prótese }\end{array}$} & \multicolumn{3}{|c|}{$\begin{array}{c}\text { Amputação Bilateral } \\
\text { Uso da prótese }\end{array}$} \\
\hline & Contínuo & Não contínuo & Não uso & Contínuo & $\begin{array}{c}\text { Não } \\
\text { contínuo }\end{array}$ & $\begin{array}{l}\text { Não } \\
\text { uso }\end{array}$ \\
\hline$\geq 80 \leq 100$ & 09 & 06 & 09 & 01 & - & 03 \\
\hline$\geq 60<80$ & 03 & - & 04 & - & 01 & 01 \\
\hline$\geq 40<60$ & - & - & - & - & - & 03 \\
\hline Total & 12 & 06 & 13 & 01 & 01 & 07 \\
\hline
\end{tabular}

Na pontuação proposta por Barthel, em anexo,100 pontos indicam que o indivíduo é totalmente independente; valores maiores ou iguais a 60 indicam alta probabilidade do indivíduo continuar a viver na comunidade; valores menores que 40 indicam importante dependência, enquanto menores ou iguais a 20 indicam aumento da mortalidade ${ }^{(6)}$.

Os idosos do presente estudo apresentaram alta capacidade funcional segundo a avaliação de Barthel. $\mathrm{Na}$ Figura 1 apresentamos a distribuição da pontuação do índice de Barthel dos sujeitos deste estudo.

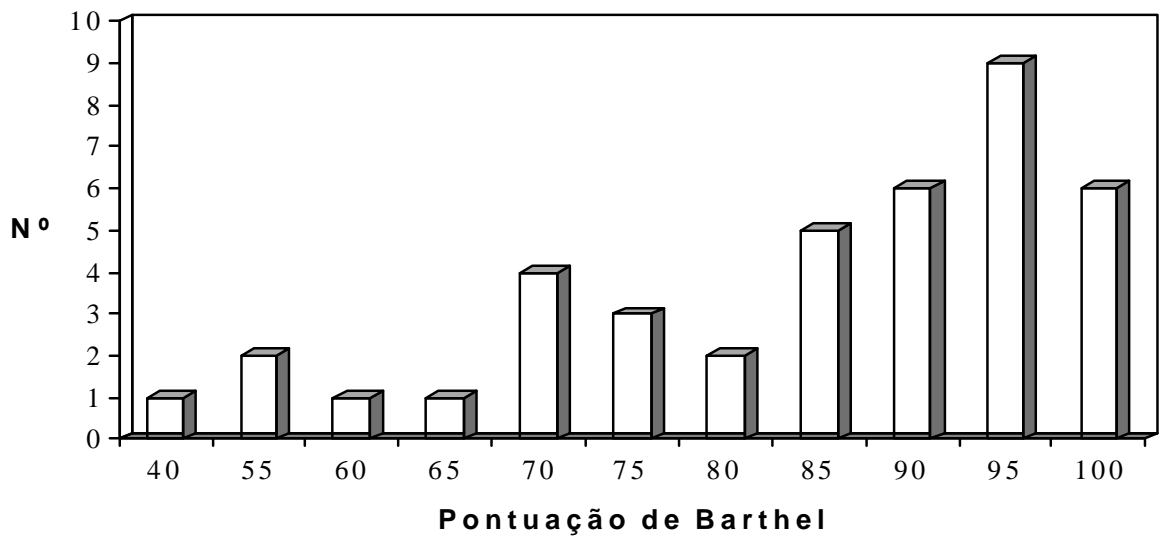

Figura 1 - Distribuição das pontuações do índice de Barthel dos idosos entrevistados. Campinas-SP, 2000 
Notamos que a maior concentração de idosos $(n=9)$ encontra-se em pontuações que traduzem elevada ou total independência, com alto desempenho funcional para as atividades avaliadas. Ainda percebemos um outlier, isto é, um indivíduo que tem o índice de Barthel muito diferente dos demais, nesse caso, muito abaixo dos outros, com pontuação igual a 40. Trata-se de uma idosa, com 76 anos de idade, submetida a amputação bilateral, portadora de diabetes mellitus e que não fazia uso de prótese. Sua pontuação indica importante dependência, segundo os critérios para o índice aplicado ${ }^{(6)}$.

No que diz respeito às dez atividades avaliadas no índice de Barthel, os resultados foram os seguintes:

- alimentação: 38 idosos eram independentes e dois necessitavam de alguma ajuda. Nenhum era dependente; - transferências: 35 idosos eram independentes, enquanto quatro necessitavam de pouca ajuda e um de muita ajuda. Nenhum era dependente total;

- toalete pessoal: 34 idosos eram independentes e seis dependentes;

- uso do banheiro: 36 idosos eram totalmente independentes, enquanto três necessitavam de ajuda e um era dependente;

- tomar banho: 30 idosos eram independentes e dez dependentes (necessitavam de alguma ajuda);

- andar em superfície plana: 26 eram independentes (conseguia caminhar no mínimo 50 metros sem ajuda, mesmo utilizando acessórios), quatro necessitavam de ajuda e dez só utilizavam cadeira de rodas. Nenhum era totalmente dependente;

- subir e descer escadas: oito idosos eram independentes, 11 necessitavam de ajuda, enquanto 21 eram incapazes de subir escadas (dependentes);

- vestir-se e despir-se: 33 idosos eram independentes, seis necessitavam de ajuda e somente um não realizava essa atividade (dependente);

- controle do esfíncter anal: 39 idosos eram independentes e somente um necessitava ajuda (apresentava acidente ocasional);

- controle do esfíncter vesical: 29 idosos eram independentes, oito necessitavam de ajuda e três eram incontinentes.

Esses resultados nos mostram que os idosos apresentavam dificuldades em maior número para as atividades de subir e descer escadas e andar em superfície plana, seguido do controle do esfíncter vesical e em menor número para tomar banho.
Ao compararmos os valores de Barthel, com o tipo de amputação e uso de prótese (Tabela 1), verificamos que dos 20 sujeitos da pesquisa que não utilizavam prótese, 12 apresentaram elevados valores de Barthel, no entanto, somente um apresentou pontuação 100 (transfemural unilateral); cinco apresentaram pontuação entre 60 e 80 e apenas três, valores inferiores a 60 (amputação bilateral).Vale destacar que, desses últimos, dois obtiveram 55 pontos e um 40 pontos.

Por outro lado, entre os idosos que utilizavam prótese ( $n=20)$, mesmo com seu uso não contínuo, 16 estavam com os níveis mais altos de Barthel, isto é, entre 80 e 100 pontos, sendo que cinco atingiram a pontuação 100 , todos esses com amputação unilateral e transtibial.

Os demais, na faixa de 60 a 80, somente quatro utilizavam a prótese (três com amputação unilateral e um bilateral).

Ao analisarmos o índice de Barthel (categorizado) com as variáveis tipo e nível da amputação e uso da prótese, utilizamos o teste exato de Fisher e obtivemos associação significativa apenas entre índice de Barthel e tipo de amputação ( $p=0,016)$, ou seja, os idosos com amputação bilateral têm Barthel menor que os idosos com amputação unilateral. Para as demais variáveis não houve associação: índice de Barthel e nível da amputação $(p=0,302)$ e índice de Barthel e uso da prótese $(p=0,456)$.

\section{DISCUSSÃO}

Ao considerarmos os dados obtidos referentes especificamente à amputação, constatamos que a grande maioria dos idosos apresentou amputação unilateral, com predomínio do nível transtibial.

Ao associarmos o nível da amputação com o desempenho funcional, avaliado por meio do índice de Barthel, verificamos que os idosos com amputação unilateral abaixo do joelho apresentaram pontuações mais elevadas (iguais e superiores a 60), indicando alta probabilidade do indivíduo continuar a viver na comunidade. No entanto, cabe destacar que a pontuação máxima não significa que o paciente possa viver sozinho, ele pode não ser capaz de cozinhar, limpar a casa, mas possui capacidade para continuar sem cuidado assistencial, pois as atividades avaliadas nesse índice são direcionadas às atividades da vida diária, que correspondem às 
necessidades básicas, com ênfase na mobilidade. Outras atividades como manutenção da casa, cozinhar, fazer compras, manusear finanças, responsabilidade com a própria medicação, utilizar o telefone e o transporte são chamadas de atividades instrumentais da vida diária, que não foram avaliadas no presente estudo.

Nos trabalhos obtidos por meio do levantamento bibliográfico, encontramos que a maioria dos idosos investigados apresentava amputação abaixo do joelho ou transtibial $^{(7-10)}$

Em função do aumento da demanda cardiopulmonar nas amputações em nível mais proximal ou bilateral, o sucesso da reabilitação com o uso da prótese, em idosos, está diretamente relacionado ao nível da cirurgia. Além disso, o tempo ocorrido entre o procedimento cirúrgico e a colocação do membro artificial está associado à maior necessidade de utilização de equipamentos acessórios para a deambulação $0^{(9)}$.

A distância, em metros, percorrida por idosos com amputação transtibial ao utilizar a prótese é maior, quando comparada ao nível transfemural, bem como a capacidade para subir e descer escadas ${ }^{(7)}$.

A recomendação da prótese para treino de marcha ocorre para aproximadamente $60 \%$ dos idosos com amputação transtibial e de 20 a $30 \%$ com amputação transfemural. Porém, somente 2 a $3 \%$ de todos os idosos com amputação são indicados para a colocação da prótese $^{(1)}$.

A observação desses autores quanto à indicação da prótese para treino de marcha é relevante, pois, em alguns serviços, a prótese pode ser indicada para o idoso somente para fins estéticos, ou seja, não utilizada para atividade de deambulação. Embora consideremos um risco para a saúde do idoso, essa situação pode ocorrer principalmente quando o mesmo apresenta alguma contraindicação clínica. No ambulatório de amputados em que atuamos, campo desta pesquisa, não é rotina a prescrição da prótese para uso cosmético, em idosos, pois o paciente pode iniciar atividades de maior gasto energético como a deambulação, sem a supervisão da equipe, correndo o risco de apresentar danos adicionais à sua saúde como infarto do miocárdio.

A prótese geralmente utilizada para amputações transtibiais é a PTB com o pé SACH (solid ankle cushion heel- tornozelo sólido e calcanhar acolchoado). Essa é um tipo de prótese que permite contato total do coto e facilita seu uso. Possui esse nome devido à capacidade de apoio do peso sobre o tendão patelar e parece constituir a melhor prótese para esse nível de amputação no momento $^{(1)}$.

Já na prótese do tipo KBM, o apoio é realizado sobre o tendão patelar e côndilos tibiais, proporcionando maior estabilidade em caso de cotos mais curtos, ou de pessoas idosas que não apresentam musculatura suficientemente desenvolvida e que necessitam de maior segurança.

Para manter a suspensão do membro artificial, muitas vezes faz-se necessário o uso de cinto ou correia acima do joelho, o que proporciona maior facilidade para a colocação e retirada da prótese, bem como maior segurança, principalmente em se tratando de pessoas com idade mais avançada.

$\mathrm{Na}$ amputação transfemural é utilizada mais comumente a prótese composta de soquete com contato total quadrilateral, com ou sem sucção, podendo ser usado um cinto pélvico, se o coto for muito proximal. O seu apoio dá-se na região do ísquio, proporcionando a impressão de realizar a marcha como se estivesse sentado sobre ela. Quanto ao joelho, vários modelos existem no mercado, como hidráulicos e de baixa energia, no entanto, sua indicação e treinamento dependem das condições do paciente e da assistência profissional. O importante é que proporcione estabilidade durante a marcha, então, para idosos, muitas vezes é indicado o joelho com trava positiva e movimento durante a fase de apoio e de oscilação da marcha, respectivamente, ou joelho que possua trava manual e de segurança autobloqueante. O pé geralmente é do tipo $\mathrm{SACH}^{(1,11)}$.

Alguns autores avaliam o desempenho funcional tendo como referência o uso da prótese na comunidade ou somente em casa.

Assim encontramos um estudo com 116 idosos, dos quais, 22 entre 30 pacientes com nível transfemural receberam prótese e treinamento. Desses, 14 utilizavam a prótese a maior parte do dia, com ou sem o auxílio de bengala (prótese funcional), ou a utilizavam parte do tempo, somente em casa, permanecendo em cadeira de rodas por longo período (prótese parcialmente funcional), após seis a 31 meses. Quanto ao nível transtibial, 59 entre 66 idosos receberam prótese e treinamento; desses, 58 foram avaliados como prótese funcional ou parcialmente funcional após o mesmo período ${ }^{(12)}$.

Em nível nacional, o trabalho realizado no centro de reabilitação da Santa Casa de São Paulo, SP, embora 
não tenha avaliado somente idosos, $42 \%$ dos pacientes eram geriátricos. Independentemente da faixa etária, ao analisarem o uso da prótese provisória, $80 \%$ dos entrevistados faziam uso da mesma, sendo que $42 \%$ em nível comunitário, 22\% no domicílio, 30\% como uso terapêutico e 6\% não conseguiram utilizá-la em decorrência de distúrbios clínicos e sensoriais que impediam a marcha $^{(13)}$.

O uso satisfatório da prótese continua baixo entre os idosos, tendo em vista que entre 69 pacientes fixados com próteses, $81 \%$ a utilizavam ao redor das suas residências, mas somente $16 \%$ haviam adquirido mobilidade mais próxima ao normal ${ }^{(14)}$.

A preservação cirúrgica da articulação do joelho favorece a deambulação com prótese e um melhor ganho funcional, principalmente em idosos. O padrão de marcha adquirido pode se aproximar do normal e a energia despendida é muito menor do que em níveis mais altos de amputação. Mesmo que o paciente não apresente condições clínicas para a deambulação com o membro artificial, seu uso pode ser indicado e favorecer as atividades de transferência, portanto, mesmo permanecendo em cadeira de rodas, o paciente pode adquirir independência no seu domicílio ${ }^{(1,15)}$.

O nível da amputação pode interferir no desempenho do paciente para a deambulação. Assim, tanto a distância em metros quanto o tempo de caminhada são maiores entre os sujeitos com amputação abaixo do joelho utilizando prótese, quando comparados aos indivíduos com amputação acima do joelho ${ }^{(9)}$.

Em outra investigação, os autores verificaram que independentemente do nível da amputação, todos os pacientes foram capazes de caminhar 50 metros utilizando prótese e um equipamento acessório para a deambulação como bengala ou muletas. Para distância igual ou superior a 100 metros, oito entre dez idosos com amputação transfemural e 16 entre 20 com nível transtibial foram capazes de caminhar. Semelhantes números de pacientes foram obtidos para as atividades de subir e descer escadas $^{(7)}$.

Em nosso estudo verificamos que na avaliação da capacidade funcional, segundo o índice de Barthel, 26 idosos deambulavam em superfície plana, no mínimo 50 metros sem ajuda, e quatro necessitavam de alguma ajuda, mas conseguiam caminhar a mesma distância. Desses, dez não utilizavam prótese, 11 a utilizavam de modo contínuo (um deles com amputação bilateral) e cinco de modo não contínuo. Em outras atividades que exigem maior mobilidade, como uso do banheiro, tomar banho e transferências, a grande maioria dos idosos era independente, lembrando que a maior dependência foi apontada para a atividade de subir e descer escadas, a qual exige importante mobilidade, equilíbrio e transferência de peso.

Interessante e abrangente investigação foi realizada sobre os fatores que influenciam o nível de atividades das pessoas com amputação de membro inferior, em que os autores buscaram identificar os problemas em áreas específicas que contribuem para a dependência dessas pessoas. O estudo contou com 131 sujeitos, com idade média de 58 anos, submetidos a amputação uni e bilateral (para efeitos de comparação, os autores distinguiram somente o nível), e utilizando próteses. Sobre o uso da prótese, $71 \%$ dos sujeitos responderam que utilizavam a prótese de modo constante, em qualquer lugar, e $60 \%$ mais do que 12 horas; enquanto $6 \%$ nunca utilizaram o membro artificial. Sobre as atividades da vida diária investigadas, os maiores problemas apresentados pelos sujeitos foram relacionados a cuidar do quintal, tomar banho e atividades de recreação, independentemente do nível da amputação. Para os sujeitos com amputação acima do joelho as maiores dificuldades diziam respeito às atividades de compras, limpeza do chão e vestir. Quanto à participação social, somente a atividade de dançar foi apontada como a de maior dificuldade para ambos os grupos, ainda que os pacientes com amputação acima do joelho tenham referido maiores problemas para participar das atividades da igreja e festas $^{(16)}$.

O uso da prótese está estreitamente vinculado ao tipo da amputação, ou seja, as dificuldades impostas pela ausência de ambos os membros inferiores limitam o uso do membro artificial, conforme constatamos entre os nove idosos entrevistados, com amputação bilateral, onde somente dois utilizavam as próteses. Ademais, houve associação significativa entre esse tipo de amputação e os valores mais baixos do índice de Barthel, indicando algum grau de comprometimento da independência para as atividades avaliadas.

Para idosos submetidos à amputação bilateral, é mais provável que a reabilitação em cadeira de rodas lhes proporcione maior independência e melhores resultados, frente ao elevado gasto energético despendido à deambulação, nesse tipo de amputação, principalmente 
em níveis transfemurais (pode chegar a 280\% de acréscimo de energia quando comparado à deambulação normal), além da presença de outros problemas de saúde. Nas amputações abaixo dos joelhos a probabilidade de uso satisfatório das próteses é maior, principalmente pela ajuda fornecida pelo membro nas transferências. Assim, ao considerar as pequenas distâncias que o idoso com amputação bilateral consegue caminhar, mesmo utilizando acessórios, o gasto energético é menor e irá adquirir maior independência se sua reabilitação for realizada em cadeira de rodas $^{(15)}$.

\section{CONSIDERAÇÕES FINAIS}

À luz dos dados obtidos, podemos dizer que os idosos com amputação de membros inferiores, sujeitos

\section{REFERÊNCIAS BIBLIOGRÁFICAS}

1. Cutson TM, Bongiorni DR. Rehabilitation of the older lower limb amputee: a brief review. J Am Geriatr Soc 1996 November; 44(11):1388-93.

2. Clark GS, Murray PK. Reabilitação do paciente geriátrico. In: Delisa JA. Medicina de reabilitação - princípios e prática. São Paulo (SP): Manole; 1992. p.475-97.

3. Ring H. La rehabilitación en los ancianos. In: Organización Panamericana de la Salud. La atención de los ancianos: un desafio para los años noventa. Washington (WC): Elias Anzola Pérez; 1994. p.279-88.

4. Diogo MJD'E. O papel da enfermeira na reabilitação do idoso. Rev Latino-am Enfermagem 2000 janeiro; 8(1):75-81. 5. Paula JAM, Tavares MCGCF, Diogo MJD'E. Avaliação funcional em gerontologia. Gerontologia 1998 junho; 6(2): 81-8.

6. Mahoney FI, Barthel DW. Functional evaluation: the Barthel Index. Md St Med J 1965 February; 14:61-65.

7. Buttenshaw P, Dolman J. The Roehampton approach to rehabilitation: a retrospective survey of prosthetic use in patients with primary unilateral lower-limb amputation. Top Geriatr Rehabil 1992; 8(1):72-8.

8. Frykberg RG, Arora S, Pomposelli FB, Logerfo F. Functional outcome in the elderly following lower extremity amputation. J Foot Ankle Surg 1998 May/June; 37(3):181-5.

9. Pohjolainen $\mathrm{T}$, Alaranta $\mathrm{H}$. Predictive factors of functional ability after lower-limb amputation. Ann Chir Gynaecol 1991; 80(1):36-9.

10. Roth EJ, Wiesner SL, Green D, Wu Y. Dysvascular amputee rehabilitation - the role of continuous noninvasive cardiovascular monitoring during physical therapy. Am J Phys Med Rehabil 1990 February; 69(1):16-22.

11. Diogo MJD'E. A consulta de enfermagem centrada nas atividades da vida: proposta de assistência ao idoso com amputação de membros inferiores. [tese]. São Paulo (SP): Escola de Enfermagem/USP; 1993. desta pesquisa, apresentam elevado grau de independência para as atividades da vida diária, segundo o índice de Barthel.

Os idosos com amputação bilateral apresentam os valores de Barthel menores que aqueles idosos com amputação unilateral.

Isto posto, sugerimos que outros estudos sejam realizados nesta linha de investigação, visando a compreensão do potencial para reabilitação de idosos submetidos a amputação de membros inferiores. Desse modo, a enfermagem pode desempenhar um papel mais ativo na resposta às necessidades individuais dos idosos portadores de incapacidades crônicas, ou seja, compreender as conseqüências da incapacidade no contexto da vida de cada pessoa, reconhecendo a reabilitação como um processo contínuo que se estende além do período que se segue à alta hospitalar.

12. Steinberg F, Sunwoo I, Roettger RF. Prosthetic rehabilitation of geriatric amputee patients: a follow up study. Arch Phys Med Rehabil 1985; 66(11):742-5.

13. Matiotti MLV, Mantovani CS, Gerstner AP. Avaliação dos pacientes amputados de membros inferiores do centro de reabilitação da Santa Casa de São Paulo. Acta Fisiátrica 1999 (edição especial); 6(3):119.

14. Campbell WB, St. Johnston JA, Kernick VF, Rutter EA. Lower limb amputation: striking the balance. Ann R Coll Surg Engl 1994; 76:205-4.

15. Buttenshaw $P$. Rehabilitation of the elderly lower limb amputee. Rev Clin Gerontol 1993; 3:69-84.

16. Medhat A, Huber PM, Medhat MA. Factors that influence the level of activities in persons with lower extremity amputation. Rehabil Nurs 1990 January/February; 15 (1): 13-8. 